\title{
Advanced Techniques and Antenna Design for Pulse Shaping in UWB Cognitive Radio
}

\author{
Lise Safatly, Mohammed Al-Husseini, Ali El-Hajj, and Karim Y. Kabalan \\ Department of Electrical and Computer Engineering, American University of Beirut, Beirut 1107-2020, Lebanon \\ Correspondence should be addressed to Lise Safatly, les12@aub.edu.lb
}

Received 12 October 2011; Accepted 24 January 2012

Academic Editor: Christos Christodoulou

Copyright () 2012 Lise Safatly et al. This is an open access article distributed under the Creative Commons Attribution License, which permits unrestricted use, distribution, and reproduction in any medium, provided the original work is properly cited.

Spectrum scarcity has emerged as a primary problem in the communications technology. The combination of cognitive radio (CR) and ultra-wideband impulse radio (UWB-IR) has been proposed to solve the shortage problem by allowing smart and adaptive spectrum management, leading to UWB-CR. In a UWB-CR scheme, secondary users are supposed to ensure interference avoidance by adaptively selecting the portions of the spectrum not being used by primary users. In this paper, three different techniques for the generation of adaptive UWB pulses are studied. The Parks-McClellan algorithm is employed, a neural network is trained, and a reconfigurable band stop filter is designed to generate an adaptive waveform with nulls at specific frequencies. Simulations, measurements, and analysis show that each generated UWB pulse has remarkable advantages in the frequency utilization, spectrum avoidance, and hardware implementation.

\section{Introduction}

With the increasing use of technology and communication, spectrum resources have become scarcer and more valuable [1]. The combination of ultra-wideband (UWB) technology and cognitive radio (CR) is an encouraging approach to ease this urgent situation [2].

UWB is characterized by a unique potential in shortrange high-data-rate wireless communications. It is mainly utilized in military applications, such as high-precision radar, life search after natural disaster, positioning, and homeland security [3]. Carried out by transmitting extremely short pulses, UWB is limited by very low energy levels. Therefore, the Federal Communication Committee (FCC) released the spectral mask on UWB emission power in 2002 [4]. UWB systems are allowed to operate in the $3.1-10.6 \mathrm{GHz}$ band without a license requirement, but under very strict transmission power limits. The equivalent isotropically radiated power (EIRP) of UWB should be below $-41.3 \mathrm{dBm} / \mathrm{MHz}$ to avoid the degradation of the legal wireless system performance.

With CR technology, unlicensed users (secondary users) are allowed to access spectrum bands licensed to primary users, while avoiding interference with them [5]. This can be achieved by the use of adaptive UWB pulses characterized by the ability to form, in their spectral masks, nulls in the bands used by existing narrow-band wireless services.

To meet these requirements, two classical UWB signal design techniques are being used: UWB pulse shaping filter design and UWB waveform optimization. Although the UWB shaping filters can achieve relatively high spectrum efficiency, they require a much high sampling rate and filter orders, thus considerably complicating the hardware structure [6]. The UWB waveform optimizing, such as genetic algorithm, is simple to implement, but it remains difficult to achieve satisfactory spectrum efficiency and meet the deep frequency null at primary users' locations [7].

Orthogonal frequency division multiplexing-based UWB multiband signal (MB-OFDM) can dynamically close the appropriate subcarrier to avoid the undesired interference. This flexible solution is limited by the attenuation depth of the spectrum notches [8]. Thus, having multiple UWB secondary users can cause a serious degradation of the legal signal due to the cumulated interferences. In [9], Yang presented a pulse based on Hermit Gaussian functions, but the need of a deep frequency null is not solved in his 
proposal. In [10], a novel antenna is designed to attenuate the transmitted signal in the primary user bands. The large size of the UWB antenna limits its applications.

Most of these techniques can only handle a fixed environment and not achieve dynamic interference cancellation, or they are complex to implement and slow to adapt to a changing environment. On the other hand, the fundamental feature of a cognitive radio is to instantly adapt to the active environment. Motivated by these requirements, we study three different techniques that generate a smart, dynamic, and adaptive UWB waveform.

The first technique relies on an advanced signal processing algorithm, Parks-McClellan (PM), which is combined with CR technology to generate an adaptive UWB pulse for multiple narrowband interference suppression. Results confirm the robustness of the generated pulse in that it closely meets the FCC mask and achieves the desired spectrum nulls. Second, a novel pulse generator based on a neural network (NN) is studied. The designed NN can adaptively modify its parameters to adjust its output spectrum according to the state of primary users. The obtained UWB pulse can maximize the spectrum utilization, and can also dynamically perform spectrum avoidance. Last, the design of a reconfigurable band stop filter is proposed. The filter is characterized by two basic elements: split-ring slots to introduce frequency notches and electronic switches to enable the control of these notches. One of the switching conditions guarantees the all-pass behavior of the filter. The remaining switching combinations result in band stops at unauthorized frequencies. The filter was integrated in the feed line of a UWB antenna which could be used to transmit the resulting adaptive pulse. Simulation and measurements illustrate the dynamic response of the designed filter and the characteristics of the antenna.

The rest of this paper is organized as follows. In Section 2, the PM algorithm is implemented. In Section 3, the design of the neural network is detailed. In Section 4, the reconfigurable filter and antenna are presented. Finally in the Conclusion Section, the performance of three techniques are compared and contrasted.

\section{The Parks-McClellan Algorithm}

2.1. Simulation Details. In this section, we are going to show our first method to generate an adaptive UWB pulse for multiple narrowband interference suppression: the ParksMcClellan algorithm combined with the cognitive radio (CR) technology.

The Parks-McClellan algorithm is generally utilized to design and implement efficient and optimal FIR filters [11]. In fact, the CR technology detects the bandwidth and the central frequency of narrowband primary users. To avoid the interference caused by unlicensed users, the ParksMcClellan algorithm will generates an adaptive pulse [12]. This is simulated in MATLAB using the firpm function. After several iterations, an optimum pulse and its corresponding spectrum is illustrated; this clearly shows the multiple notches at some frequencies. The inputs of the firpm function

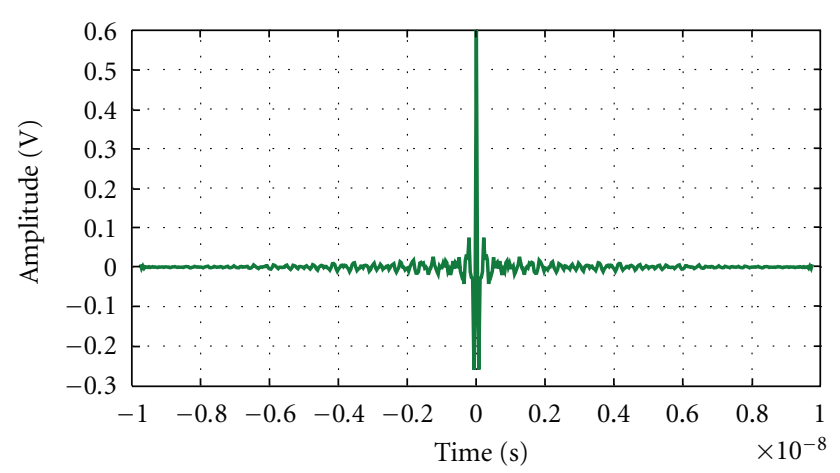

FIgUre 1: The pulse generated by the PM algorithm.

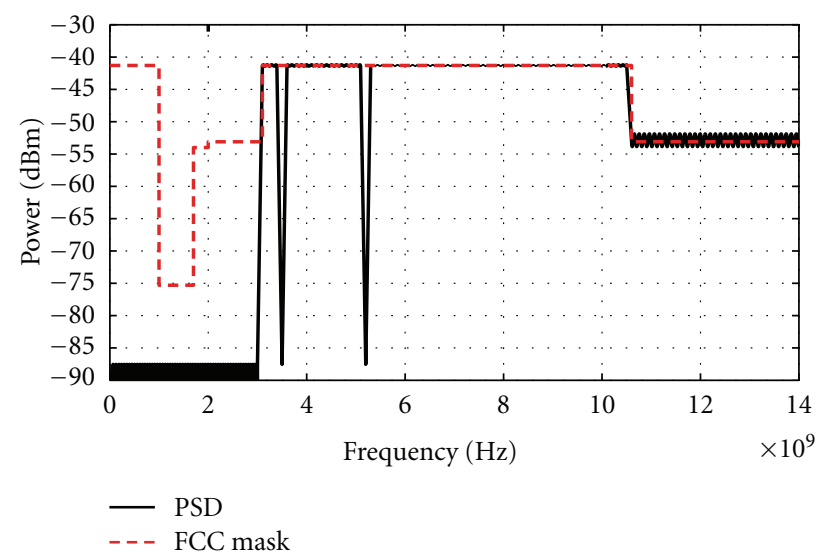

FIGURe 2: PSD of the pulse generated by the PM algorithm.

should respect the locations of the frequency notches and the FCC spectral mask. The firpmord function is used to obtain these inputs and to specify the order of the filter.

2.2. Generated Pulses. Figure 1 shows the pulse obtained by simulating the Parks-McClellan algorithm in MATLAB, considering two frequency nulls at $3.5 \mathrm{GHz}$ and $5.2 \mathrm{GHz}$, which are the famous bands of WiMAX and WLAN. The elapsed time of this simulation is 4.9 seconds, and the needed filter order is 546 . In Figure 2, the corresponding spectrum is illustrated, showing the notches.

With the help of spectrum detection technology the Parks-McClellan algorithm can generate adaptive pulses that meet the FCC spectral mask and avoid narrowband interferences. Despite the high filter order, PM algorithm is provides excellent spectrum utilization and a depth of $-45 \mathrm{~dB}$ at undesired bands.

\section{Neural Network}

3.1. Description of the Technique. The second proposed method for UWB pulse shaping is the usage of neural networks. They are composed of simple elements operating in parallel which are inspired by biological nervous systems [13]. As in nature, the network function is largely determined by the connections between elements. A neural network 


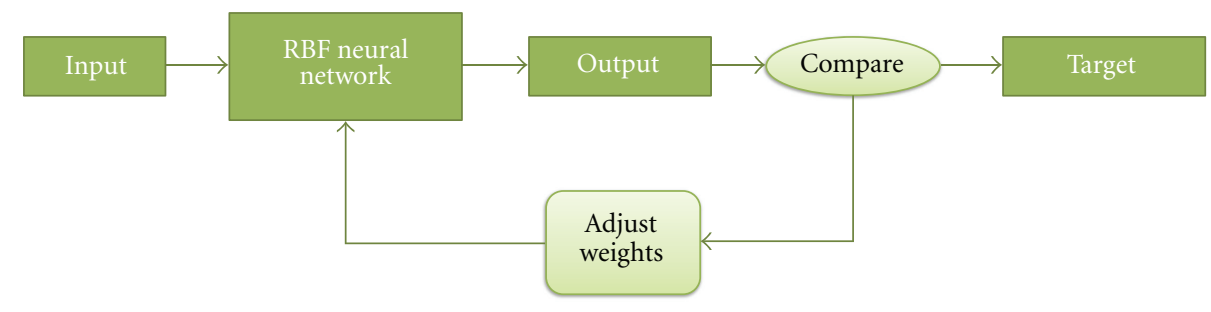

FIGURE 3: RBF neural network model.

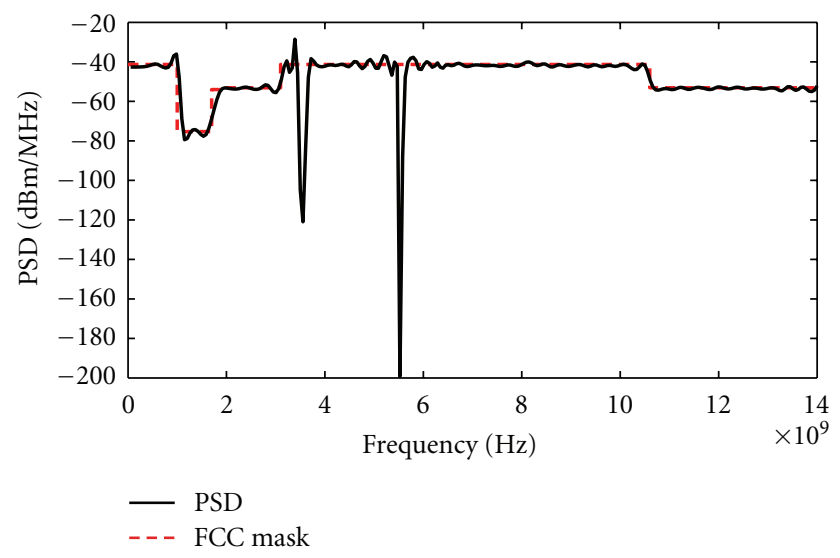

FIGURE 4: PSD of the pulse generated by the neural network in MATLAB.

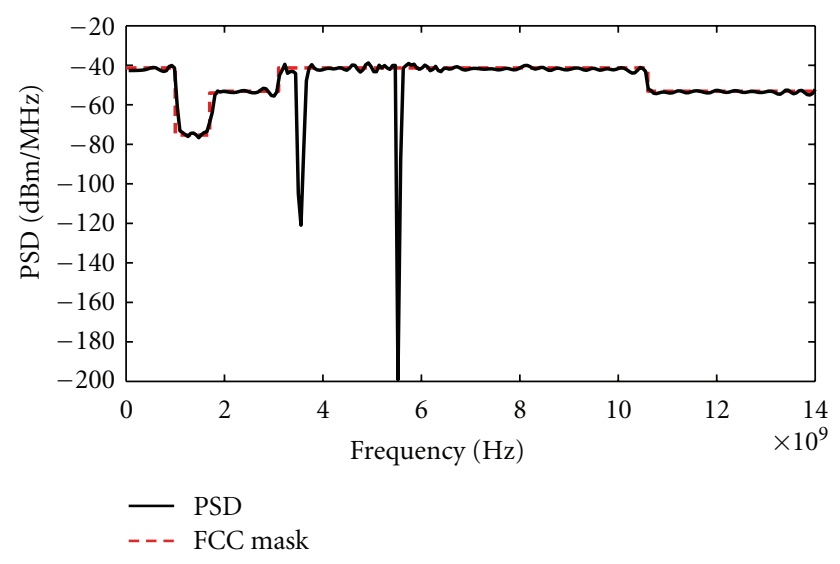

FIGURE 5: PSD of the pulse generated by NeuroSolutions.

could be designed to perform a particular function by adjusting the values of the connection (weights) between elements. In this section, this method is used to generate the dynamic UWB pulse.

Commonly, neural network are adjusted or trained so that a particular input leads to a specific target output. The network will then adjust its parameters, based on a comparison of the output and the target, until the output matches the target. The target signal is a result of spectrum sensing, which will be determined by the current frequency bands of the primary users. Then, by adjusting the network weights according to specific learning rules, the actual output

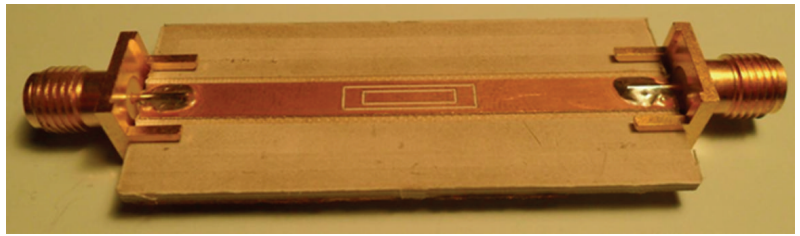

Figure 6: Photo of the designed dual stop-band filter.

can trace the target signal after a very limited number of iterations.

The radial basis function network will be selected in this paper because it has the capability to ensure the requirements suggested previously. It is shown that RBF network can approximate any function at any precision by altering its basis functions [14]. Figure 3 shows a simplified block diagram to explain our model.

The RBF network needs an input matrix $P$ based on a basis function $p(n)$ that is supposed to meet two requirements as follows.

(1) The basis function should be even symmetry around its center.

(2) The energy of the basis function should be finite.

Based on above restrictions, a famous candidate for the basis waveform is the Gaussian function [15]. In this case, the total network input $P$ can be written as

$$
P=\left[p_{0}, p_{1}, p_{2}, \ldots, p_{n 2}\right]^{T} .
$$

The basic Gaussian function is given by

$$
\begin{gathered}
p_{0}(k)=1 \quad k=0,1, \ldots, N-1, \\
p_{i}(k)=\frac{1}{\sqrt{2 \pi} s_{i}} \exp \left(-\frac{f_{s}^{2}}{2 s_{i}^{2}}\left(k-\mu_{i}\right)^{2}\right) \quad i>1, k=0,1, \ldots, N-1 .
\end{gathered}
$$

3.2. Simulations Results. The input matrix is formed using the basis waveform $p(n)$. The input vectors $p_{i}$ are generated using MATLAB. To obtain two different networks and compare their results, two types of software are used in our paper.

First, the neural network Toolbox in MATLAB is utilized. During the learning step, the network weight vector is updated. After the network weights have achieved its convergence, the learning process will be terminated. Finally, 


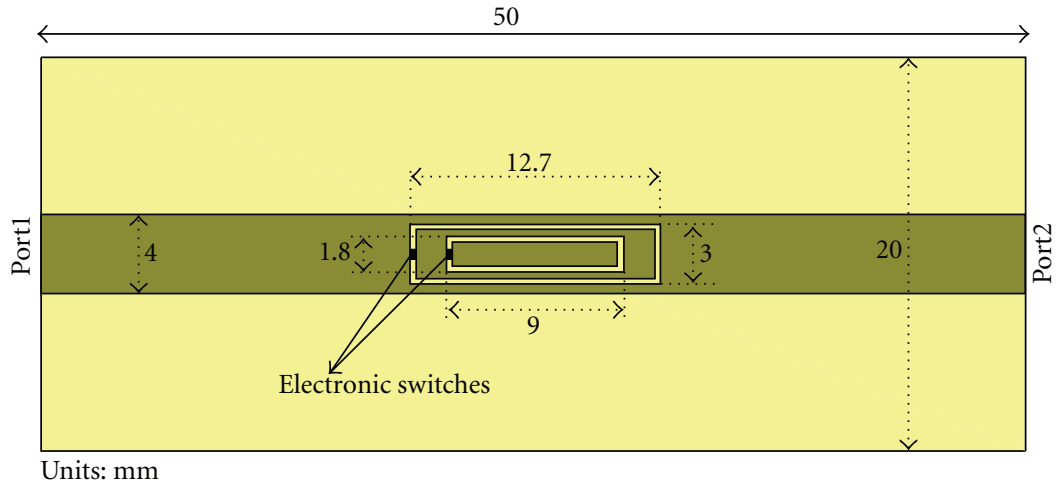

FIgURE 7: Configuration of the designed dual stop-band filter.

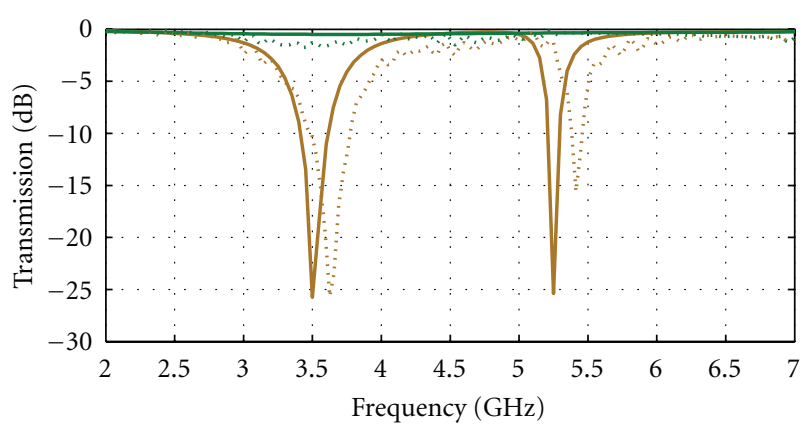

— Simulated: filter On …... Measured: filter On — Simulated: filter OFF ….. Measured: filter OFF

FIGURE 8: Simulated and measured S11.

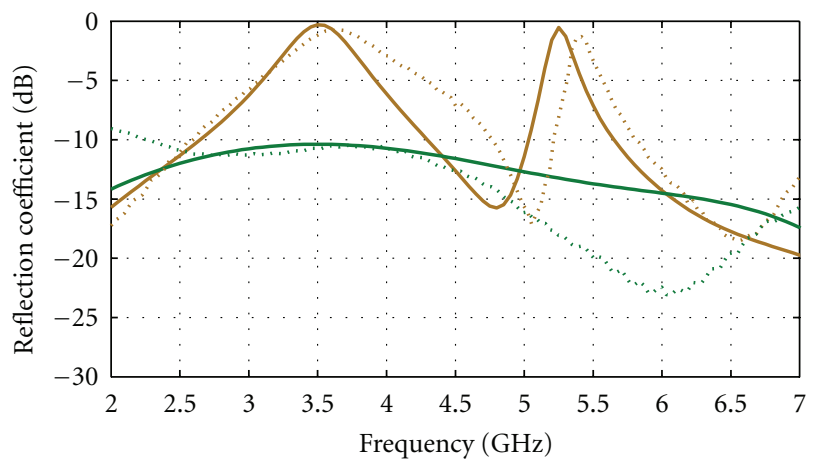

— Simulated: filter On $\quad \cdots . .$. Measured: filter On — Simulated: filter OFF $\quad \cdots \cdots$ Measured: filter OFF

FIgURE 9: Simulated and measured S21.

the transmit pulse is derived from the network output. The target signal can be dynamically determined by the result of spectrum sensing. In the simulation, we will consider the FCC mask with the two notches at WiMAX and WLAN frequency locations. Our goal is to optimize the learning time to ensure a dynamic system. The PSD of the obtained pulse using the Neural Network training tool in MATLAB with 31 iterations is shown in Figure 4.

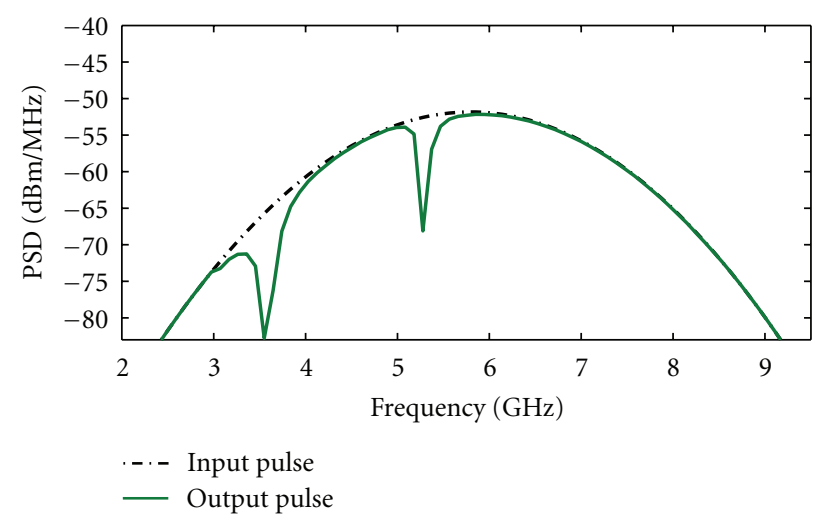

FIGURE 10: Input Gaussian monocycle and corresponding output.

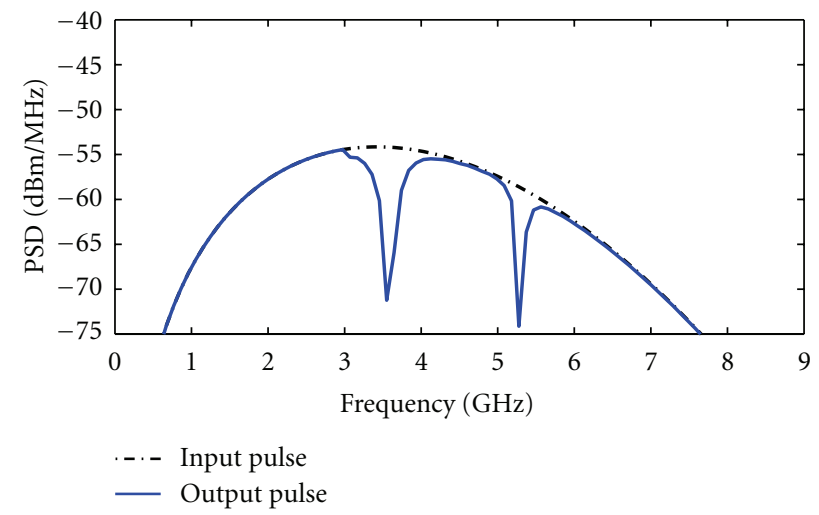

FIGURE 11: Input Scholtz's monocycle and corresponding output.

Second, same simulation parameters are used in "NeuroSolutions": the premier neural network development Environment. The same input (based on Gaussian functions) and the same target signal (FCC mask with 2 frequency nulls at $3.5 \mathrm{GHz}$ and $5.2 \mathrm{GHz}$ ) are given. The PSD of the obtained results using NeuroSolutions is shown in Figure 5 with a number of iterations equal to 120 .

The proposed UWB pulse generation using neural networks provides much safer protection to the primary users. 


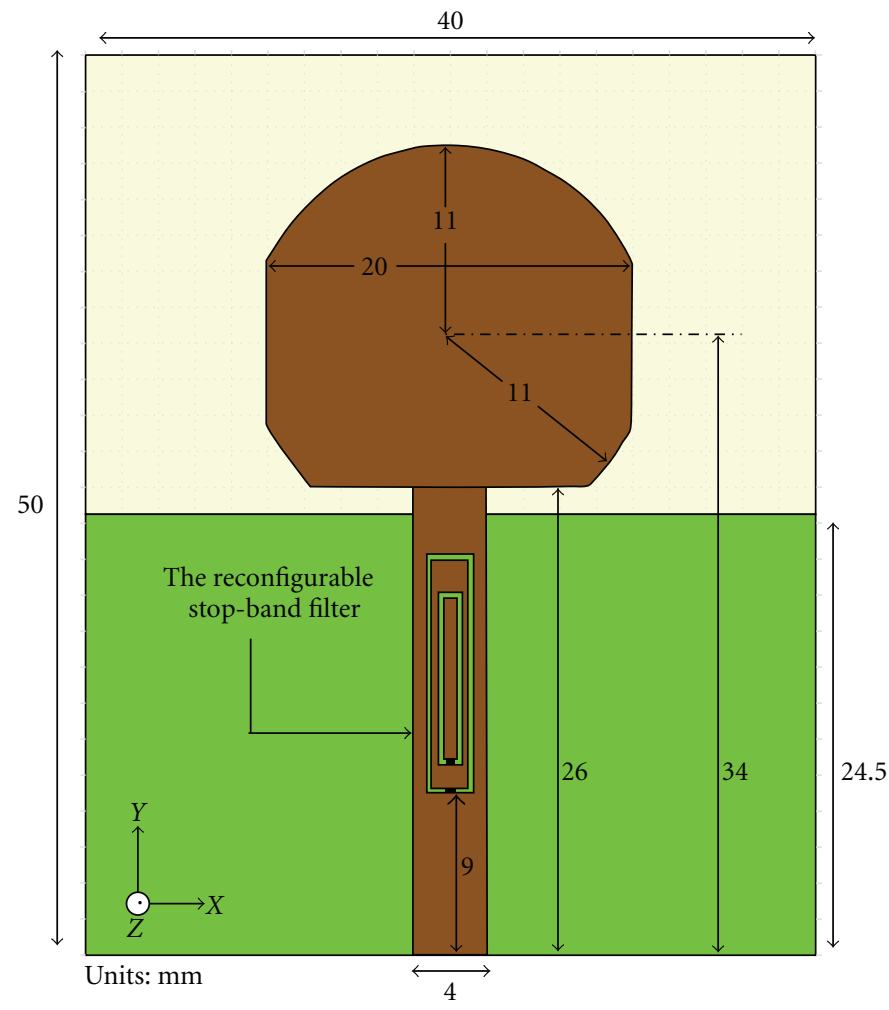

FIgURE 12: Antenna geometry.

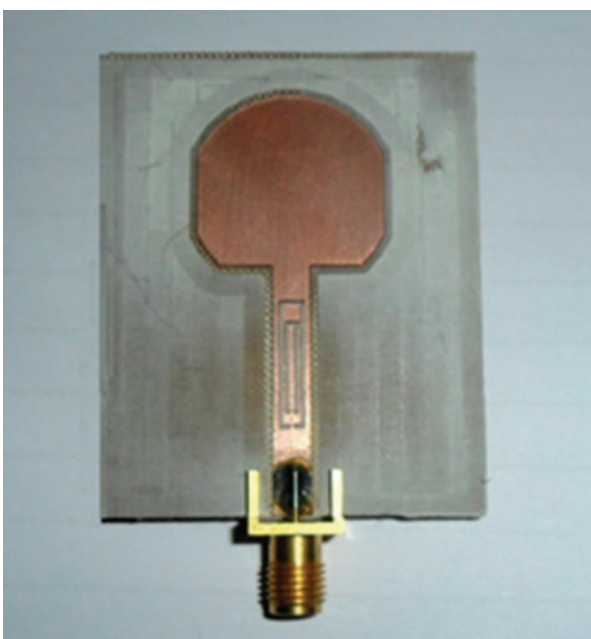

Figure 13: Antenna photo.

When the number of iterations of the learning step is increasing, the generated pulse becomes more efficient ensuring perfect frequency utilization and spectrum avoidance since it provides the deepest frequency notch.

\section{Filter Antenna Design}

The last technique investigated in this paper is based on a filter-antenna design. A reconfigurable stop-band filter is

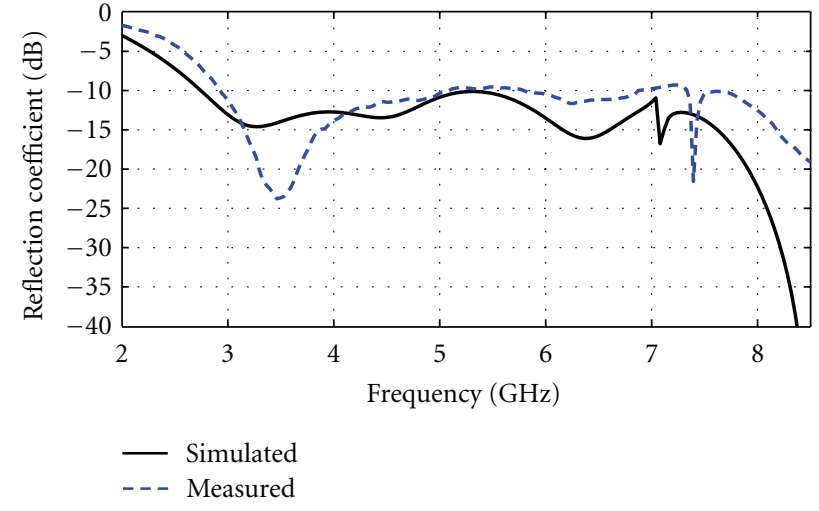

FIGURE 14: Reflection coefficient when all switches are tuned OFF.

firstly designed, and then it is incorporated on the feed of a UWB antenna to ensure pulse shaping and interference avoidance with licensed users.

4.1. Filter Design and Stop-Band Control. A dual stop-band filter is shown in Figure 6. The filter is a $50 \Omega$ microstrip line, printed on a $1.6 \mathrm{~mm}$ thick Rogers RO3203 substrate with $\varepsilon_{r}=3.02$, on which two rectangular split-ring slots have been incorporated with two electronic switches mounted across each ring slot. The split-ring slot is a single-ring complementary split-ring resonator (CSRR). CSRRs and their dual-counterparts, the SRRs [16], have been employed in [17] for the design of UWB antennas with fixed notched 


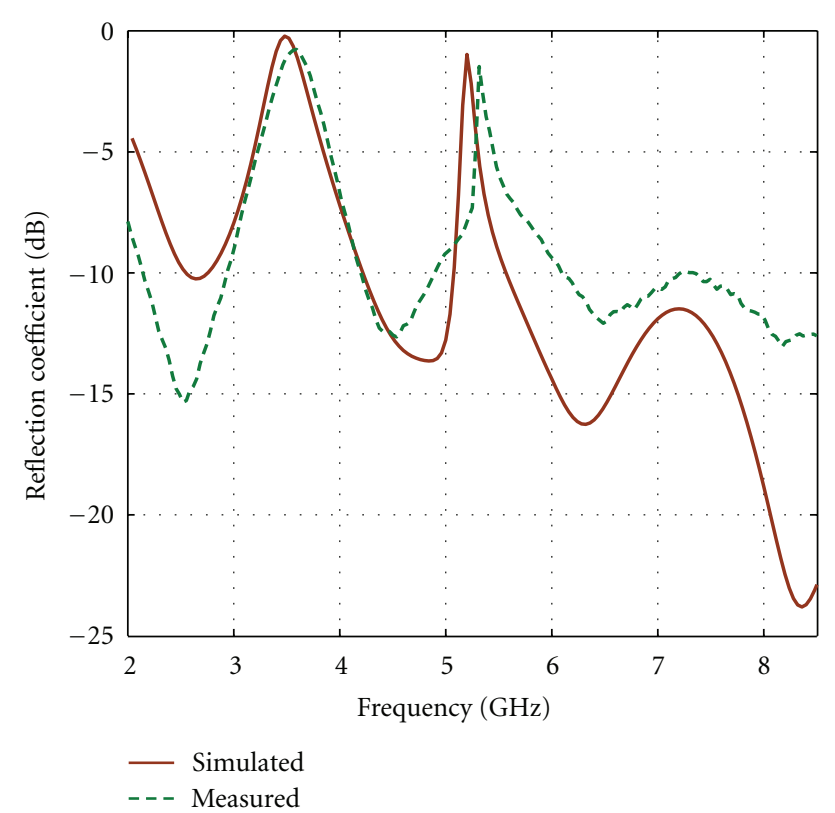

FIGURE 15: Reflection coefficient when all switches are turned ON.

bands and in [18] for the design of UWB antennas with multiple controllable band notches.

The dimensions of the CSRRs are indicated in Figure 7. If the electronic switch is $\mathrm{ON}$, the corresponding CSRR will resonate inducing a band stop in the primary user's operation band. In our designed filter, CSRRs are parameterized to cause nulls at $3.5 \mathrm{GHz}$ and $5.2 \mathrm{GHz}$. To avoid other wireless systems, further band stops could be generated by adding other CSRRs. If no primary users are active, the filter should reestablish its all-pass behavior by turning OFF all the switches.

4.2. Measured and Simulated Characteristics of the Filter. The filter is simulated using Ansoft HFSS [19]. A prototype of the filter is fabricated, so the reflection coefficient (S11) and transmission (S21) are simulated and measured. Although the structure of the filter is not perfectly symmetrical, the simulations and measurements have shown that S11 and S22 are very close. The same property applies to S21 and S12. Figures 8 and 9, respectively, show the computed and measured S11 and S21 plots. Two of four switching cases are illustrated: the all-pass behavior and the dual-stop bands. It is shown that when both switches are ON, two stop-bands occur at 3.5 and $5.2 \mathrm{GHz}$. Generating only one band stop is also possible by turning OFF one of the switches. The plots show good analogy between simulated and measured results.

4.3. Generated Pulses. A UWB pulse will be inputted to the filter the output from the other filter port will be also a UWB pulse, with very similar shape, except in band stops, where the pulse will have nulls. These nulls help to avoid interference to primary users or services operated in these bands.

In UWB communications information is contained in narrow pulses (monocycles). Several possible monocycles could be utilized. In this paper, two types of UWB pulses are tested with the filter: Gaussian and Scholtz's monocycles. The Gaussian monocycle is similar to the first derivative of the Gaussian pulse and is given by [20]

$$
W(t)=2 \times \frac{A}{\tau} \times \sqrt{e} \times\left(t-T_{c}\right) \times \exp \left[-2 \times\left(\frac{t-T_{c}}{\tau}\right)^{2}\right] .
$$

In (3), $\mathrm{A}$ is the amplitude, $\tau$ the pulse shape parameter, and $T_{c}$ the time duration of the pulse.

On the other hand, Scholtz's monocycle is an attractive waveform because of its flat power spectrum density, which best suits the designed filter. This monocycle is similar to the second derivative of the Gaussian pulse and can be represented by [21]

$$
W(t)=A\left[1-4 \pi\left(\frac{t-T_{c}}{\tau}\right)^{2}\right] \exp \left[-2 \pi\left(\frac{t-T_{c}}{\tau}\right)^{2}\right] .
$$

Figure 10 shows the PSD of the inputted Gaussian monocycle and its corresponding output pulse. Similarly, Figure 11 shows that for Scholtz's monocycle. Both figures reveal an attenuation of about $15 \mathrm{~dB}$ in the filter's stop-bands.

4.4. Antenna Configuration and Results. In this section, an antenna suitable for UWB-CR is proposed. Having our designed filter integrated on its feed line, the proposed antenna is reconfigurable in terms of the ability to selectively have band stops in the WLAN and WiMAX bands.

Depicted in Figure 12, the antenna is a monopole printed on a $1.6 \mathrm{~mm}$ thick Rogers RO3203 substrate with $\varepsilon_{r}=3.02$, and features a partial rectangular ground plane. The patch is almost circular in shape. The dimensions of the different parts are optimized for an impedance bandwidth covering the UWB range (starting $3 \mathrm{GHz}$ ). To induce band notches, the reconfigurable stop-band filter is integrated in the feed line. Switching cases studied in Section 4.2 are also applicable on the antenna.

The antenna was designed and simulated using Ansoft HFSS [19]. A prototype was fabricated and illustrated in Figure 13, and the reflection coefficient was measured for two operation scenarios. The all-pass behavior is showed in Figure 14 and two band stops at $3.5 \mathrm{GHz}$ and $5.2 \mathrm{GHz}$ are added in Figure 15 by turning ON the switches. The HFSScomputed normalized gain patterns in the $\mathrm{X}-\mathrm{Z}$ and $\mathrm{Y}-\mathrm{Z}$ planes, for each case, are shown in Figure 16. All cases result in omnidirectional patterns. The patterns are unchanged for the switches-ON and switches-OFF cases. This means that outside the rejected bands, the patterns are independent of the filter state.

\section{Conclusion}

In this paper, we studied three different techniques to generate adaptive UWB waveforms for application in UWBCR. Two of these techniques, Parks-McClellan and neural network, are based on advanced signal processing algorithms. The third relies on the design of reconfigurable RF 


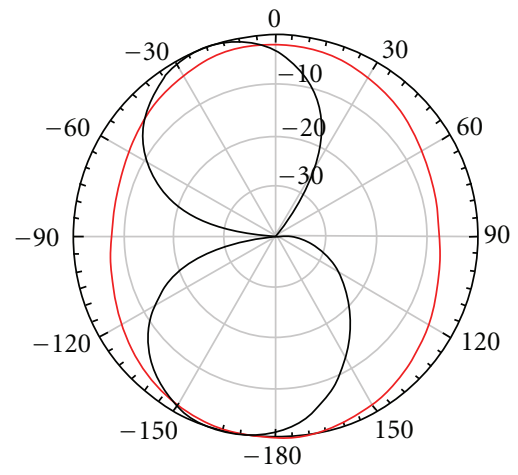

4.5 GHz-switches ON

(a)

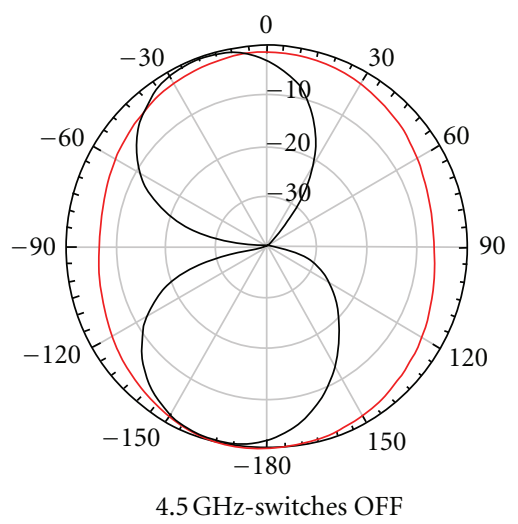

(c)

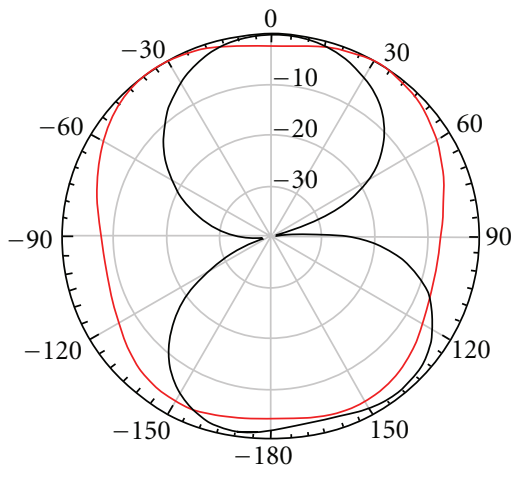

$6 \mathrm{GHz}$-switches $\mathrm{ON}$

(b)

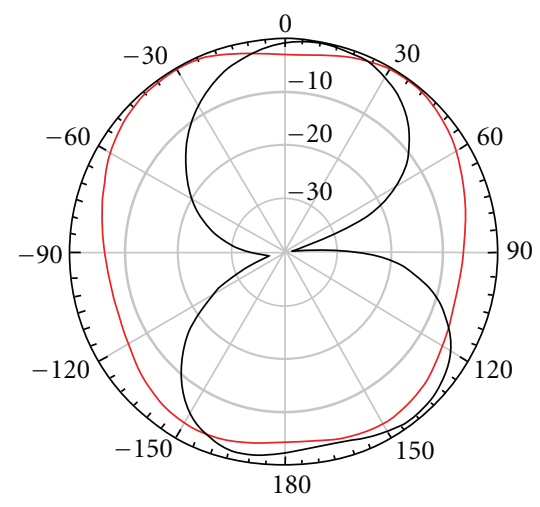

$6 \mathrm{GHz}$-switches OFF

(d)

FIGURE 16: Radiation pattern at 4.5 or $6 \mathrm{GHz}$ when switches are turned ON or OFF.

filters. MATLAB simulations showed that the PM algorithm can achieve high spectrum utilization. The main drawback of this technique is its hardware complexity resulting from the high filter order. On the other hand, neural networks, which are easier to implement to fit any practical function, can be used as a UWB-adaptive pulse generator. Our simulated neural network provided a UWB waveform overcoming all the challenges of UWB-CR after a limited number of iterations. The reconfigurable stop-band filter was easier to implement and had the advantage of simple integration in the feed line of a UWB antenna. Simulated and measured results for the filter and the antenna were presented. Despite its advantages, the nulls generated by this technique were not as deep as those of the first two techniques.

\section{References}

[1] Z. Zhou, B. Li, and Q. Song, "Open spectrum and UWB research in China: spectrum reform and radio revolution," in Proceedings of the IEEE International Conference on UltraWideband (ICUWB '10), pp. 1-5, Nanjing, China, September 2010.

[2] H. Arslan and M. Sahin, "UWB-based cognitive radio networks," in Cognitive Wireless Communication Networks, pp. 213-230, Springer, New York, NY, USA, 2007.
[3] S. Nag, M. A. Barnes, T. Payment, and G. W. Holladay, "An ultra-wideband through-wall radar for detecting the motion of people in real time," in Radar Sensor Technology and data Visualisation, vol. 4744 of Proceedings of SPIE, pp. 48-57, Orlando, Fla, USA, 2002.

[4] Federal Communications Commission (FCC), "Revision of part 15 of the commission's rules regarding ultra-wide band transmission systems," Tech. Rep. ET Docket 98-153, FCC 0248, 2002.

[5] K. C. Chen and R. Prasad, Cognitive Radio Networks, John Wiley \& Sons, West Sussex, UK, 2009.

[6] D. S. Zeng, A. Annamalai, and A. I. Zaghloul, "Pulse shaping filter design in UWB system," in Proceedings of the IEEE Conference on Ultra-Wideband Systems and Technologies (ICUWB-ST'03), pp. 66-70, Reston, Va, USA, 2003.

[7] S. Liao, M. Ho, C. Chen, C. Chiu, and W. Chung, "A novel DS-UWB pulses design using genetic algorithm," in Proceedings of the International Conference on Future Computer and Communication, pp. 296-300, Kuala Lumpur, Malaysia, 2009.

[8] M. P. Wylie-Green, "Adjacent frequency coding technique for decreasing MB-OFDM ultra-wideband interference to other radio services," in Proceedings of the IEEE Sarnoff Symposium (SARNOF '06), pp. 1-4, Princeton, NJ, USA, 2006.

[9] R. Yang, Z. Zhou, L. Zhang, and C. Yu, "Detection and avoidance scheme for direct-sequence ultra-wideband system: 
a step towards cognitive radio," IET Communications, vol. 2, no. 8, pp. 1043-1050, 2008.

[10] T. Dissanayake and K. P. Esselle, "Prediction of the notch frequency of slot loaded printed UWB antennas," IEEE Transactions on Antennas and Propagation, vol. 55, no. 11, pp. 3320-3325, 2007.

[11] J. H. McClellan and T. W. Parks, "A personal history of the Parks-McClellan algorithm,” IEEE Signal Processing Magazine, vol. 22, no. 2, pp. 82-86, 2005.

[12] X. Shi, "Adaptive UWB pulse design method for multiple narrowband interference suppression," in Proceedings of the IEEE International Conference on Intelligent Computing and Intelligent Systems (ICIS '10), vol. 1, pp. 545-548, Saint Louis, Mo, USA, 2010.

[13] J. J. Hopfield, "Neural networks and physical systems with emergent collective computational abilities," Proceedings of the National Academy of Sciences of the United States of America, vol. 79, no. 8, pp. 2554-2558, 1982.

[14] J. D. Powell, "Radial basis function approximations to polynomials," in Numerical Analysis, pp. 223-241, Longman, Dundee, UK, 1987.

[15] L. Bin, Z. Zheng, and Z. Weixia, "A novel spectrum adaptive UWB pulse: application in cognitive radio," in Proceedings of the IEEE 70th Vehicular Technology Conference Fall (VTC '09), pp. 1-5, Anchorage, Alaska, USA, September 2009.

[16] J. B. Pendry, A. J. Holden, D. J. Robbins, and W. J. Stewart, "Magnetism from conductors and enhanced nonlinear phenomena," IEEE Transactions on Microwave Theory and Techniques, vol. 47, no. 11, pp. 2075-2084, 1999.

[17] Y. Zhang, W. Hong, C. Yu, Z. Q. Kuai, Y. D. Don, and J. Y. Zhou, "Planar ultrawideband antennas with multiple notched bands based on etched slots on the patch and/or split ring resonators on the feed line," IEEE Transactions on Antennas and Propagation, vol. 56, no. 9, pp. 3063-3068, 2008.

[18] M. Al-Husseini, A. Ramadan, Y. Tawk, C. Christodoulou, K. Kabalan, and A. El-Hajj, "A planar ultrawideband antenna with multiple controllable band notches for UWB cognitive radio applications," in Proceedings of the 5th European Conference on Antennas and Propagation (EUCAP '11), pp. 375-377, Rome, Italy, 2011.

[19] Ansoft HFSS, Pittsburg, Pa, USA.

[20] P. Withington, Impulse Radio Overview, Time Domain Corporation.

[21] X. Chen and S. Kiaei, "Monocycle shapes for ultra wideband system," in Proceedings of the IEEE International Symposium on Circuits and Systems, pp. I/597-I/600, May 2002. 

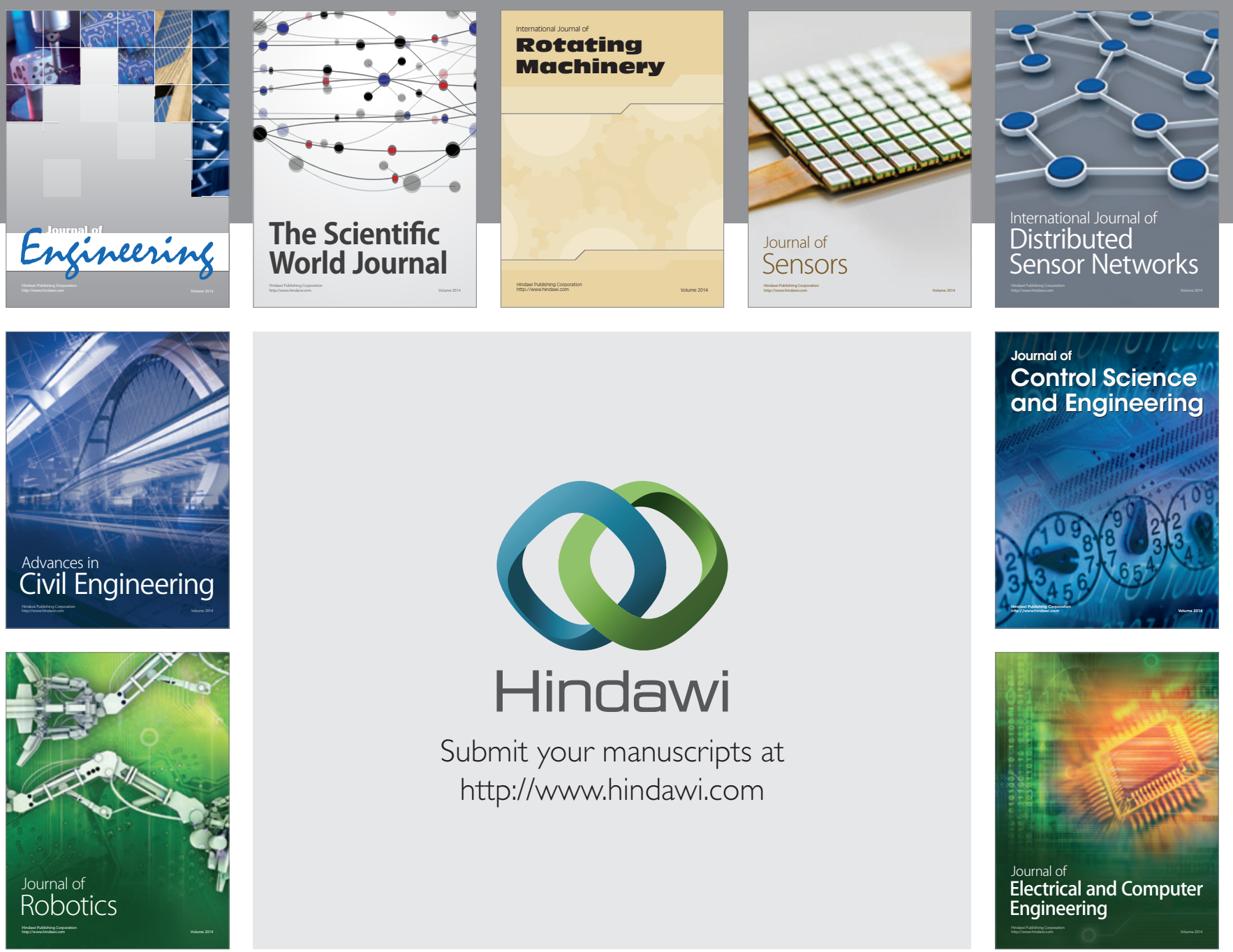

Submit your manuscripts at

http://www.hindawi.com
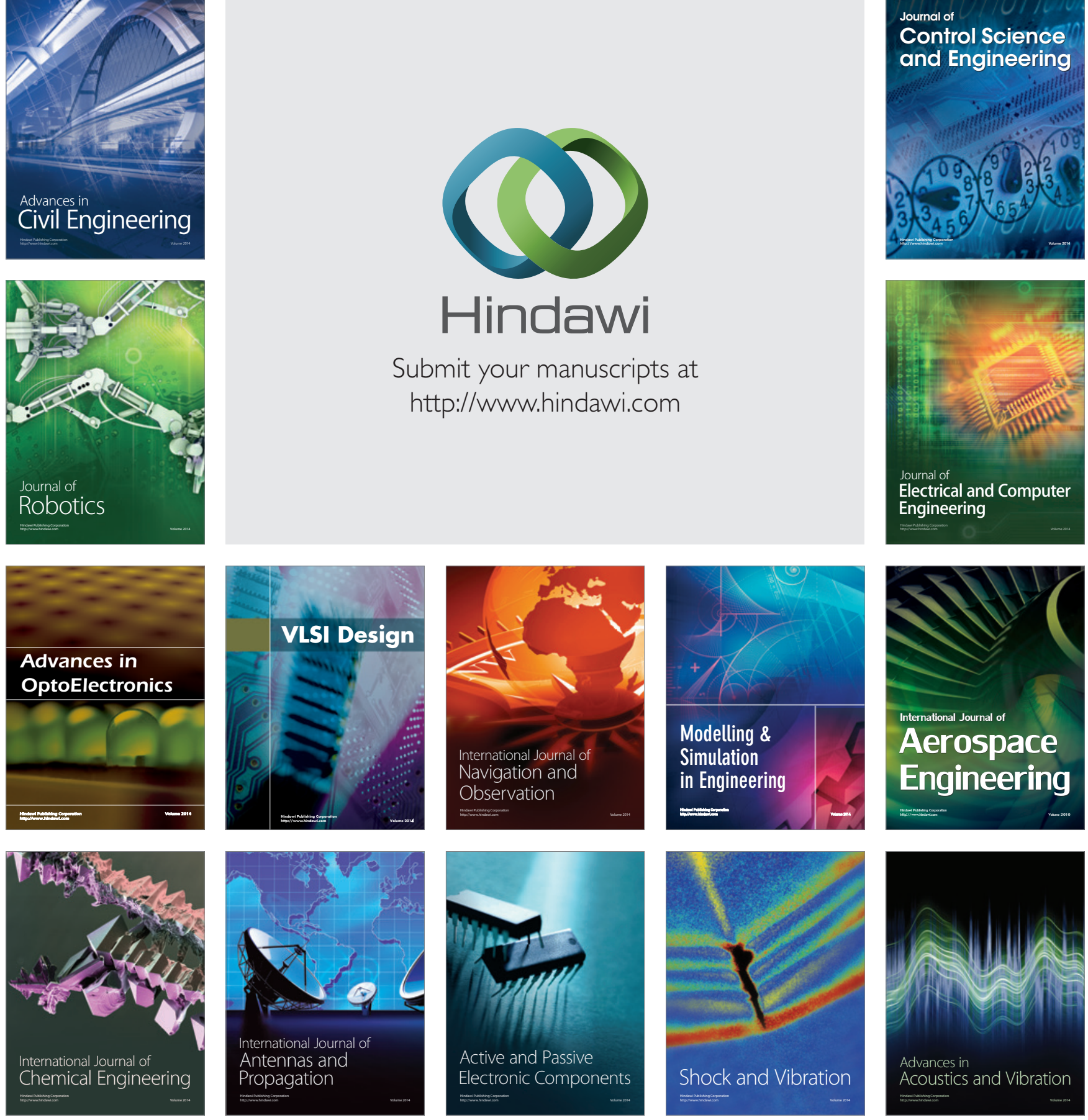